\title{
Internet of Things Online Monitoring System Based on Cloud Computing
}

\author{
https://doi.org/10.3991/ijoe.v13i09.7591 \\ Kehua Xian \\ Sichuan College of Architectural Technology, Sichuan, China \\ 3117827 eqq. com
}

\begin{abstract}
In order to develop a new convenient online monitoring system for Internet of things, an online monitoring system based on cloud computing is designed. The performance of this new Internet of things technology used in modern agricultural is test by Amazon relational database service (RDS) and ZigBee perception network. By analyzing the Internet of things related technologies and agricultural modernization, the integration framework of the Internet of things, cloud computing and data mining technology in the field of modern agriculture are proposed. Through the modern agricultural Internet of things monitoring system, the Internet of things intelligent gateway, cloud based research and construction of large data analysis and data mining projects are verified. The experimental results show that the relevant parameters of the model are obtained by training about $70 \%$ of the original data after adopting the cloud computing. Based on the above finding, it is concluded that the open Internet of things platform needs to be supported by the powerful computing resources. In addition, the cloud computing technology is suitable for the development of the Internet of things service platform.
\end{abstract}

Keywords - Internet of things; cloud computing; modern agriculture

\section{Introduction}

Modern agriculture refers to the social agriculture with modern science and technology, the means of production and scientific management methods provided by modern industry. The development of modern agriculture mainly consists of two aspects. On the one hand, it is to equip agriculture with advanced science and technology and production factors so as to realize the modernization of the means and techniques of agricultural production [1]. On the other hand, it is to realize the specialization, socialization, regionalization and industrialization of agricultural production through the modernization of agricultural organization management [2]. In order to realize the modernization of agricultural production and organization management, the introduction of Internet technology has become inevitable. In this regard, the realization of agricultural modernization has made considerable progress in the adoption of technologies such as Internet of things, cloud computing, and large data analysis [3]. 
In modern agricultural production, the perception equipment of Internet of things technology is used to collect the perception data such as light intensity, environmental temperature and humidity, soil $\mathrm{pH}$ value, soil fertility and crop growth status. After a series of processing, the data is uploaded to the support layer of Agricultural Internet of things cloud platform through the network layer. Through the use of cloud computing technology for real-time analysis of the perceived data, the Internet of things cloud platform will make intelligent decision-making feedback [4]. This process has realized the real-time monitoring of agricultural production and the automatic control of agricultural production equipment, so that the crops are always in a good and suitable growth environment [5]. Agricultural production has gradually shifted from a production model centered on manpower to an isolated machine centered on information and software. Through the precise control of agricultural use, water use, fertilizer use, and the precise control of livestock and aquaculture, agricultural waste and pollution are reduced. Disease prevention and epidemic prevention and control are also gradually strengthened, which promote the agricultural production and realize the meticulous management of modern agriculture. Agricultural Internet of things and cloud computing technology can also be applied to the logistics, transportation and traceability of agricultural products. This process has realized the management of agricultural products from production, processing, warehousing, transportation, distribution and sales [6]. It not only ensures food safety, but also promotes the intensification, scale and standardization of agricultural products.

\section{$2 \quad$ Literature review}

Since twenty-first Century, some countries such as China, Europe and the United States have carried out the application research of Internet of things in the agricultural field. Some achievements have been made in the fields of agricultural resources utilization, agricultural ecological environment monitoring, agricultural production and agricultural product safety supervision. At the same time, it promoted the development of related information industry and its standardization [7].

In recent years, in the field of agricultural resources monitoring and utilization, some countries in Europe and America, as well as in China, have gradually established a relatively complete monitoring and utilization system of agricultural resources. These new systems can better realize the monitoring of crop growth, soil moisture, agricultural natural disasters, agricultural meteorological parameters and agricultural ecological environment. In Hawaii, for example, agriculture is the mainstay of its economy. But the shortage of water resources has been a threat to the local agricultural development. The state government has built up an advanced farming irrigation system through the use of Internet of things technology [8]. Projections of rainfall have been carried out by satellite data. Through the Internet of things and data fusion technology, the whole process of water resources rational allocation and transportation process is realized, thus realizing the monitoring and rational utilization of water resources on the island. 
In the field of agricultural ecological environment monitoring, some countries in Europe and America have established a monitoring platform for agricultural ecological environment which can cover the whole country by using sensing technology, Internet technology and information fusion technology, ensuring the sustainable development of agricultural production [9]. For example, almost every state in the United States has established a fairly perfect monitoring system of water, soil, air resources and environment, and realized the real-time detection of agricultural ecological environment [10]. Some European and American countries such as the United States, Australia and other countries have applied extensive fine management measures in the fields of crop planting, precision operation, facilities, agricultural environmental monitoring and irrigation and fertilizer control [11]. For example, many farmers in Australia use remote sensing satellites to monitor the growth of crops. Through the geographic information system (GIS) and satellite positioning (GPS) technology, the efficient cooperation of combine harvester is realized. In our country, a series of application systems have been developed in the fields of soil environment information acquisition, agricultural equipment perception, data collection and automatic control, and good results have been achieved [12].

\section{Methods}

\subsection{Requirement analysis of system intelligent gateway}

The Internet of things gateway is an integral part of the Internet of things application system. As a link between connection aware network and traditional communication network, it needs access to a variety of cognitive networks. The ability to convert data between different protocols, access to the Internet quickly, and the ability to control and manage perception devices are required.

1. Perceptual network access: The perceptual network is an important part of the implementation of perceptual data acquisition. The gateway of the Internet of things needs to have the access ability of the sensing network, and collect the sensed data acquired by the sensing network, which acts as the coordination node role of the sensing network. It has the interface to communicate with the nodes of the sensing network, and then realizes the connection with the whole perception network.

2. Multiprotocol data conversion: The protocols used in the Internet of things gateways and various sensory networks are not the same. The essence of two-way data exchange between the gateway of the Internet of things and the perception network is to realize the conversion between various transport protocols. The sensed data collected by the sensing network is sent to the gateway. The gateway parses the data and then packages it into packets to be transmitted to the Internet of things cloud platform via the internet.

3. Rapid access to the Internet: The Internet of things connects the perceived network to the Internet, enabling the perception of data from the perceptual network. The value cloud service platform is delivered via the Internet, enabling connectivity be- 
tween the perceived network and the internet. As a result, Internet users are able to see the status of devices running in the gateway at any time.

\subsection{Perception network based on Zigbee}

ZigBee is a low power LAN protocol based on the IEEE802.15.4 standard. ZigBee is translated as "violet bee", which is similar to bluetooth. According to international standards, ZigBee technology is a wireless communication technology with shortrange and low-power. It is characterized by close distance, low complexity, selforganization, low power consumption and low data rate. It is mainly suitable for automatic control and remote-control field, and can be embedded in various devices. As a short-range wireless communication technology, ZigBee network can provide wireless data transmission for users conveniently, so it has very strong applicability in the field of Internet of things.

\subsection{Video surveillance based on motion detection}

In our modern agriculture network monitoring system, a video surveillance module is designed. Through video surveillance, users can understand the growth of crops in real time, and monitor the actual operation of the Internet of things equipment. With the help of raspberry branch gateway's rich expansion ability and strong computing power, remote real-time video surveillance is successfully realized by using USB high-definition camera and open source software motion. Combined with the simple head made by stepping motor, the function of remote control rotating camera is realized.

\section{$4 \quad$ Results and discussion}

\subsection{Large data analysis based on Cloud Computing}

In the Internet of things applications, the data generated is massive. If the Internet system has 10000 sensors to collect data every $5 \mathrm{~S}$, then the system will be able to reach hundreds of billions of orders of data one year only in sensor data. Dealing with such a large amount of data will be a major problem for the system. One of the basic functions of the system is to provide users with real-time sensing, data query and statistical functions. It is not advisable to query and analyze data directly from a large amount of data for each request, which will take up a large amount of disk, IO and computing resources. At the same time, the delay is not acceptable. As a result, the Hadoop large data analysis hosting platform service EMR (Elastic MapReduce) provided by Amazon has been adopted. As shown in figure 1, the EMR timed operation is used to periodically analyze and calculate large data in the DynamoDB database. The result is transferred to the partition table of Oracle. It can improve the quality and response speed of the system service by Providing data query service to users through Oracle. 


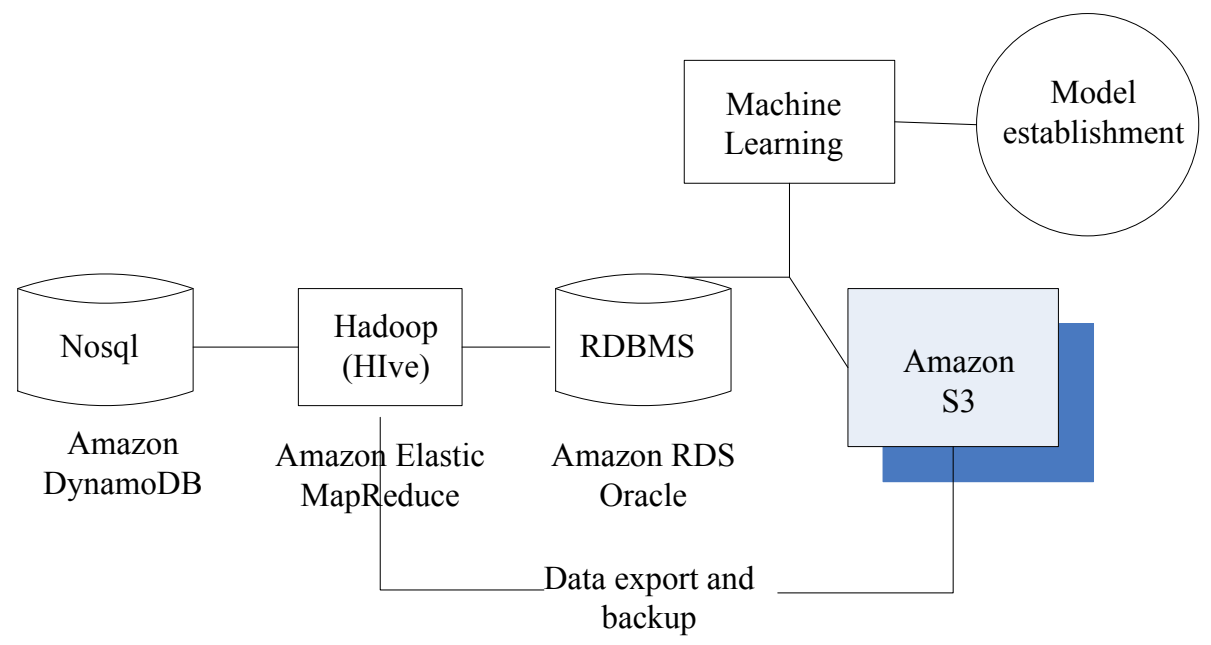

Fig. 1. System large data analysis and machine learning process

A typical example of large data analysis in the system is the average hourly value of the perceived data stored in the NOSQL database DynamoDB. When users want to query the data movement of their perception device over the past 48 hours, the system should not query all the data points within 48 hours to the user. Because the number of data generated by such queries might exceed 100 thousand. But if the system returns the average value per hour after data analysis, then it's up to 48 data points, which is sufficient for viewing data trends.

\subsection{Architecture analysis of basic software}

Raspberry faction gateway operating system uses Debian based Raspbian and the Linux Kernel Technology (the core of the operating system). But most of the underlying operating system tools come from the open source GNU project, which is also known as GNU/Linux. It was the first Linux release using a package management system, making installation and removal of software easier. Debian GNU/Linux comes with more than 29000 packages, and the precompiled software is wrapped up in a good format for installation on the computer.

In addition to using open source operating system, the system gateway also uses a lot of open source software or library files. For gateway hardware IO, I2C, SPI, the RPi.GPIO WiringPi is used. PySerial is used to monitor motion based video. In addition, many modules of the system, such as python, shal and modules provided by HTTP are used. The entire architecture of the gateway is shown in figure 2 : 


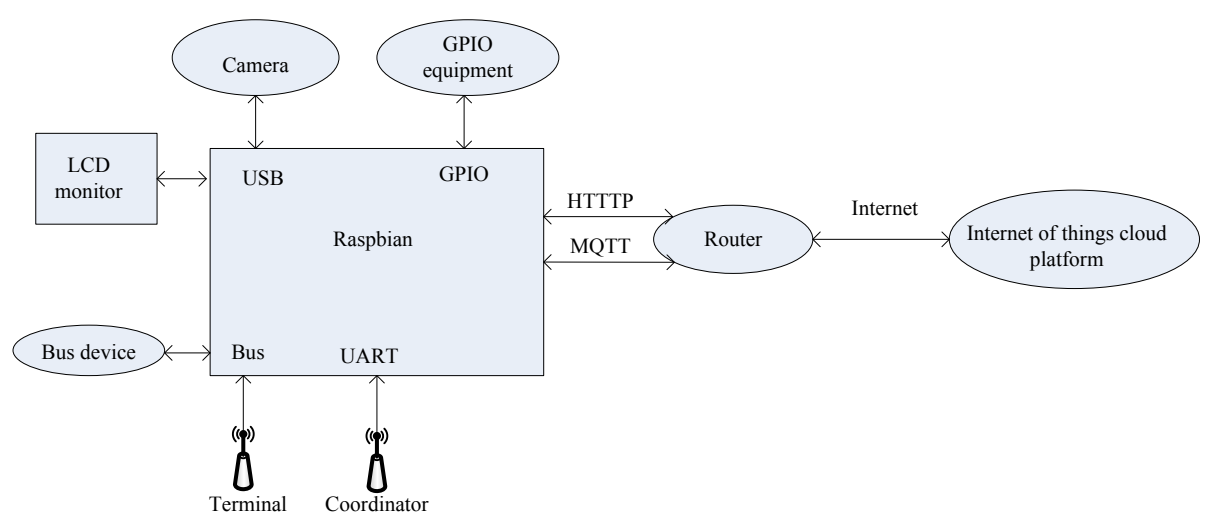

Fig. 2. The overall structure of the gateway

\subsection{Analysis of monitoring system of Internet of things in modern agriculture}

The modern agricultural Internet of things monitoring system is developed on the J2EE platform. The system needs to be run on an application server that supports J2EE. The common ones are paid WebSphere, WebLogic, open source, free Jboss and Tomcat. Through the comparison of these application servers, open source, free resources, low occupancy and high concurrent Jboss are used as the application servers of the system. In the Internet of things applications, there will be very high concurrent connection requests. Therefore, Nginx is used as a front-end web server to separate static and dynamic resources, and realize the load balancing of the system using its reverse proxy function. Since the functional modules of the system are based on the session mechanism, it is necessary to consider the implementation of multiple Jboss instances while implementing system load balancing. With the high scalability of EC2, our hardware and software resources can be adjusted at any time according to the system load level, so as to ensure the normal operation of the system. The deployment structure of the whole system is shown in figure 3 . 


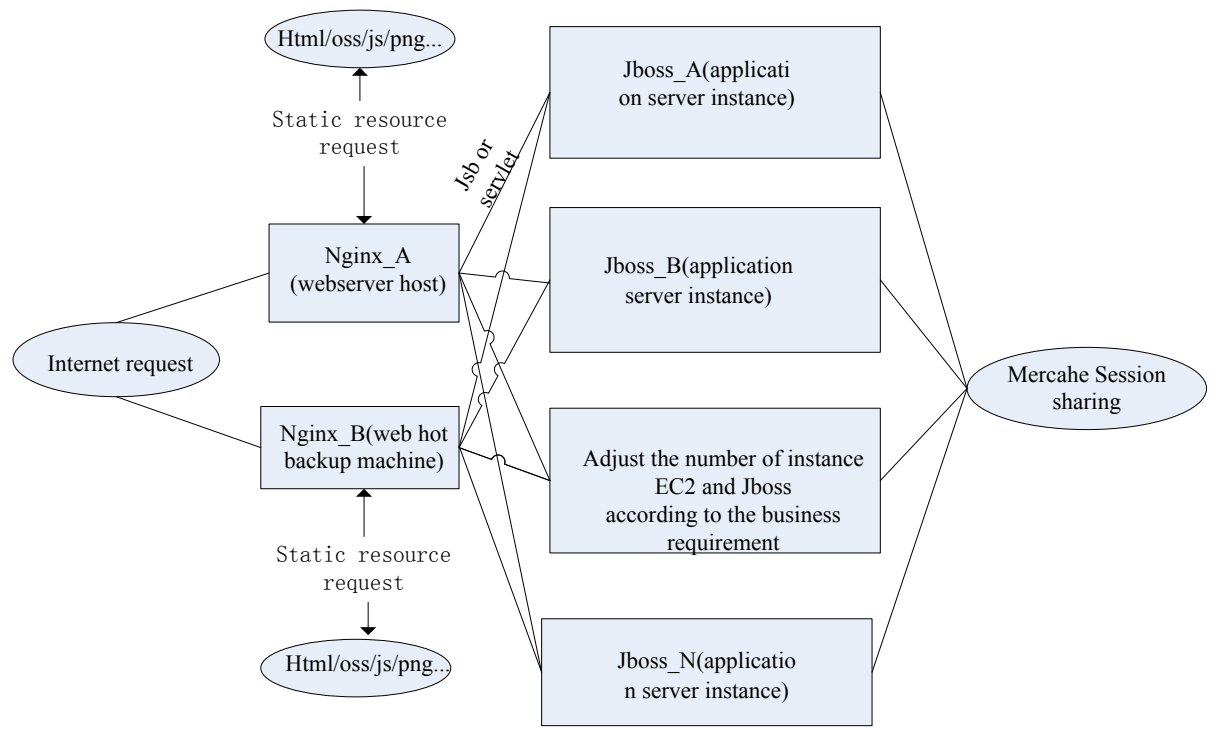

Fig. 3. System deployment diagram

\subsection{Experimental result}

Cloud computing technology is the cornerstone of the development of the Internet of things. It promotes the development of Internet of things mainly from two aspects. First of all, cloud computing is the core of the Internet of things, and it is possible to use cloud computing model to make real-time dynamic management and intelligent analysis of all kinds of objects in the Internet of things. RFID, wireless sensor networks and other new technologies are fully used in the industry through the Internet of things. It connects all kinds of objects, and gathers and analyzes and processes all kinds of real-time dynamic information collected by Internet through the Internet of things support platform.

In our modern agricultural Internet of things monitoring system, the system will be built into an open agricultural Internet of things service platform, providing data storage, large data analysis and processing functions. At the same time, the mqtt message service platform has been established to realize the transmission of messages in the manner of security, stability and low resource occupation. The Internet of things platform belongs to the support layer in the layered structure of Internet of things. Based on this platform, users can develop low cost, safe and stable agricultural networking applications with large data analysis capabilities. In order to realize these functions, the open Internet of things platform needs powerful computing resources to support. Therefore, cloud computing technology is used to develop the Internet of things service platform. 


\section{Conclusion}

In view of the coming big data era, combined with the Internet of things, cloud computing, big data and other technologies, the demand for agricultural modernization has been brought into deep application research. Through the modern agricultural Internet of things monitoring system, the Internet of things intelligent gateway, cloud based research and construction of large data analysis and data mining projects are verified. The main achievements are as follows: First, the demand and future direction of agricultural modernization in big data era are analyzed. Second, the scientific idea of combining Internet of things, cloud computing and big data with modern agriculture is put forward. Then, the overall framework of the modern agricultural Internet of things monitoring system based on cloud computing is designed and constructed. Third, open source hardware tree is used to develop a low-cost, stable and extensible intelligent gateway for Internet of things. By integrating the ZigBee module, wireless sensor networks are constructed, which successfully realize video surveillance based on motion detection, acquisition and uploading of perceptual data and remote control of equipment. Finally, the public cloud service is regarded as the basic hardware and software platform of the modern agricultural Internet of things monitoring system. The use of IAAS and PAAS services provided by cloud vendors reduces the difficulty of system development, and makes the system more stable and low cost.

Although the cloud based Internet of things online monitoring system proposed in this paper is very innovative, there are still many improvements. First, according to the design concept of the modern agricultural Internet of things monitoring system based on cloud computing, the function of the system needs to be improved. Second, several exemplary applications should be constructed to push the application of animal networking in agriculture. After accumulating enough data of Agricultural Internet of things system, the modeling method of relevant functional requirements is further studied and tested to promote the application of large data analysis in agriculture.

\section{References}

[1] Gui, L., Ruan, T. Y., Wang, Z. Z., Sun, A. C., \& Xu, M. (2015). Cnc online monitoring system based on internet of things. Advanced Materials Research, 1079-1080(6), 672-678.

[2] Abawajy, J. H., \& Hassan, M. M. (2017). Federated internet of things and cloud computing pervasive patient health monitoring system. IEEE Communications Magazine, 55(1), 48-53. https://doi.org/10.1109/MCOM.2017.1600374CM

[3] Xia, M., Li, T., Zhang, Y., \& Silva, C. W. D. (2016). Closed-loop design evolution of engineering system using condition monitoring through internet of things and cloud computing. Computer Networks the International Journal of Computer \& Telecommunications Networking, 101(C), 5-18. https://doi.org/10.1016/j.comnet. 2015.12.016.

[4] Cho, H., Kim, K. T., Jang, Y. H., Kim, S. H., Kim, J. S., \& Park, K. Y., et al. (2015). Development of load profile monitoring system based on cloud computing in automotive. , 43(4), 573-588. https://doi.org/10.7469/jksqm.2015.43.4.573. 
[5] Zhang, F., Liu, M., Zhou, Z., \& Shen, W. (2016). An iot-based online monitoring system for continuous steel casting. IEEE Internet of Things Journal, 3(6), 1355-1363. https://doi.org/10.1109/JIOT.2016.2600630

[6] Sajid, A., \& Abbas, H. (2016). Data privacy in cloud-assisted healthcare systems: state of the art and future challenges. Journal of Medical Systems, 40(6), 1-16. https:/doi.org/10.1007/s10916-016-0509-2. https://doi.org/10.1007/s10916-016-0509-2

[7] Al-Ayyoub, M., Jararweh, Y., Daraghmeh, M., \& Althebyan, Q. (2015). Multi-agent based dynamic resource provisioning and monitoring for cloud computing systems infrastructure. Cluster Computing, 18(2), 919-932. https://doi.org/10.1007/s10586-015-0449-5

[8] Ma, Y. W., Cho, W. T., Chen, J. L., Huang, Y. M., \& Zhu, R. (2015). Rfid-based mobility for seamless personal communication system in cloud computing. Telecommunication Systems, 58(3), 1-9. https://doi.org/10.1007/s11235-014-9869-4

[9] Shariff, F., Rahim, N. A., \& Hew, W. P. (2015). Zigbee-based data acquisition system for online monitoring of grid-connected photovoltaic system. Expert Systems with Applications, 42(3), 1730-1742. https://doi.org/10.1016/j.eswa.2014.10.007

[10] Yang, X., Luo, H., Krueger, M., Ding, S. X., \& Peng, K. (2016). Online monitoring system design for roll eccentricity in rolling mills. IEEE Transactions on Industrial Electronics, 63(4), 2559-2568. https://doi.org/10.1109/TIE.2015.2442223

[11] Allalou, A. N., Tadjine, M., \& Boucherit, M. S. (2016). Online monitoring and accident diagnosis aid system for the nur nuclear research reactor. Turkish Journal of Electrical Engineering \& Computer Sciences, 24(3), 1604-1614. https://doi.org/10.3906/elk-1401-272

[12] Bo, L., Hui, Y., Li, T., \& Huang, D. (2016). Study on food information online monitoring system utilized wireless sensor network. Advance Journal of Food Science \& Technology, 11(2), 137-142. https://doi.org/10.19026/ajfst.11.2368

\section{$7 \quad$ Author}

Kehua Xian is with Sichuan College of Architectural Technology, Sichuan, China (3117827@qq.com).

Article submitted 13 August 2017. Published as resubmitted by the author 19 September 2017. 